\title{
Quadruple Co-Infections due to Leptospira, Dengue, Hepatitis E \& Round Worm: A Window to Urban Insanitary
}

\author{
Aparna Patange ${ }^{1}$, Sumit Chaudhari ${ }^{2}$, B Kiran Babu ${ }^{2}$, Bhavik Shah ${ }^{2}$ \\ ${ }^{1}$ Assistant Professor, Department of Internal Medicine, KIMS, Karad \\ ${ }^{2}$ Post Graduate Residents, Department of Internal Medicine, KIMS, Karad
}

\begin{abstract}
Co-infection in a immune competent host is rare. Leptospirosis, Dengue, Hepatitis E and Ascariasis are diseases commonly transmitted in locales with poor hygiene and sanitation and overcrowding. Not too many cases of quadruple co-infection in an immune competent host have been reported. We report a case of mixed infection due to leptospira, dengue, hepatitis $E$ and Ascariasis. Like clinical presentation and simultaneous transmission of these diseases, especially during the monsoon often leads to a dilemma in diagnosis. Early detection of the disease by appropriate laboratory tests and timely institution of therapy is pivotal and life saving.
\end{abstract}

Keywords: Co-infection; Leptospira; Dengue; Hepatitis E ; Round worm

\section{Introduction}

Leptospirosis is a worldwide zoonosis caused by Spirochetes that almost affects 160 mammalian species, however, rodents are the most important resorviors. Two main species of spirochetes are identified: Pathogenic L interrogans and free-living $L$ biflexa. The pathogenic variety is further classified into 250 serovars belonging to 25 different serotypes. The disease is particularly endemic in areas of heavy rainfall combined with poor hygiene and sanitation facilities and over crowding. Clinical features of Leptospirosis range from mild anicteric illness to a lifethreatening icteric illness ( Weil's Syndrome ). It potentially involves different organ systems including the Liver, Kideneys, Lungs and the Brain and can cause lifethreatening haemodynamic instability. The true incidence of leptospirosis in India is not known since it is underdiagnosed and under-reported due to the lack of the awareness about the disease \& relative unavailability of appropriate diagnostic facilities in most parts of the country. IgM ELISA is the most widely used rapid diagnostic test for detection of antibodies.

Dengue, caused by flavivrus is one of the most important mosquito-borne arboviral infections of humans. Large Indian cities are its hyper-endemic zones. Infection is caused by any one of the four closely related serotypes of the virus designated Dengue virus 1-4. The infection usually causes a non-specific acute febrile illness, dengue fever (DF); however, in few cases it is characterized by plasma leakage causing dengue shock syndrome (DSS) \& a life threatening Dengue Haemorrhagic Fever (DHF) in few other cases. In India, latest dengue epidemic was reported from Delhi in 2006. Detection of Dengue IgM antibody is the most widely used serological test. RT-PCR is gold standard but impracticable.

Hepatitis E is a common water-borne infection hyperendemic in India, specially in regions of dismal sanitation facilities. It can be considered zoonotic as serological evidence of the virus has been found in the feces of pigs, sheep and cattle. It presents as an acute febrile illness with jaundice. It is selflimiting and requires no more than an adequate nutritional support. Fulminant hepatic failure and death occurs in about $0.1 \%$ of the cases. Diagnosis is by detection of Anti HEV IgM in the serum. Prolonged prothrombin time is common, however this is hardly a cause of bleeding. There is direct hyperbillirubinemia with 5 fold increase in AST and modest rise in ALT.

Ascariasis ( Round Worm ) is the most common helminthic infection endemic in India. It is transmitted by feco-oral route. The disease remains asymptomatic in most cases but can also present more fulminantly as Obstructive jaundice, pulmonary haemorhage or intestinal obstruction. The diagnosis is by demonstration of the round worm ova in the stool microscopy. Some time adult round worms are also detected incidentally on an Abdominal Ultrasound.

These diseases have been routienly discussed individually. But, here, we report a case of mixed infection due to Leptospira, Dengue, HEV \& Ascariasis while we try to press the urgency for urban sanitation.

\section{Case Report}

A 23-year-old gentlemen, a salesman from Mumbai, presented to the medicine outpatient department of Krishna Hospital, Karad, with a history of fever and generalized weakness for 7days, jaundice for 5 days, pain abdomen for 5 days \& vomiting for 3 days. There was no rash or bleeding from any site. The fever was continuous and was not associated with chills, rigors or headache. It was relieved by drugs that he received from a GP. The abdominal pain was dull and dragging and was localized to the right hypochondrium. The vomiting was non-bilious, nonprojectile, 1-2 episodes per day and was associated with nausea.The patient is a non-alcoholic. There was no history of loose stools. There was no remarkable drug history nor a history of blood transfusion. On general examination, the 


\section{International Journal of Science and Research (IJSR) \\ ISSN (Online): 2319-7064 \\ Index Copernicus Value (2013): 6.14 | Impact Factor (2014): 5.611}

patient was conscious \& oriented, there was fever, $101^{\circ} \mathrm{F} \&$ Icterus . BP was 130/60 mmHg. On systemic examination, there was a right hypochondriac tenderness and guarding with mild hepatomegaly, $2 \mathrm{~cm}$ below the right costal margin. There was no ascites or splenomegaly. The examination of respiratory system and CVS revealed no abnormalities.

Based on the history and clinical examination, a provisional diagnosis of Acute viral hepatitis was considered. Also, a differential diagnosis of Malaria, Enteric fever, Leptospirosis and Dengue fever was not ruled out. Further, initial investigations revealed deranged liver functions (Total billirubin : 5.0mg/dl Direct 2.6, Indirect 2.4, AST : $>2000$ IU/L, ALT : 338IU/L, ALP : 125 IU/L. ) with a deranged coagulation profile ( Prothrombin time : 23secs, INR 2.33 ). Renal Function tests were normal ( Urea : 28, Creatinine : 0.9 ). Routine hematological investigation was normal including the platelets count. However, a few leucocytes showed toxic changes with reactive lymphocytosis. Based upon this lab data, a diagnosis of Acute Viral hepatitis was made and conservative treatment initiated. Meanwhile investigations to establish a definitive etiology for hepatitis were ordered. Peripheral blood smear for Malarial parasite and the WIDAL test for enteric fever were negative. $\mathrm{HbSAg}$ and anti-HCV antibodies were also negative. A routine USG was performed which incidentally revealed multiple round worms which was further confirmed on the Stool microscopy after which the patient was treated with Albendazole. Considering the nature of the patients job and his exposure to Mumbai floods, the patient was investigated for Leptospirosis. Rapid test for Leptospira IgM antibodies came out to be positive. Hence, the patient was given Injection Ceftriaxone $2 \mathrm{gm}$ i.v OD which was continued for a period of 7 days. Concurrently, in view of high endemicity and high prevelance of Dengue especially during monsoons in the Mumbai suburbs and the platelets counts on a decreasing trend [ 1.50 lacs/cu $\mathbf{~ m m ~ - ~} 1.05$ lacs/cu $\mathbf{~ m m}$ in 3 days ], the patients serum was tested for dengue specific IgM antibodies which showed positive results. During the period of hospital stay, there was no mark deterioration in the patients general condition, however there was a progressive derangement of liver functions. On day 8 , considering the patient's rising bilirubin levels [5.0 mg\% (on day 1) - 12.7 mg \%( on day 8)], predominantly conjugated hyperbilirubinaemia and worsening INR [ 2.3 ( on day 1 ) - 3.0 ( on day 7 ) ], the patient was investigated for hepatitis E. Anti-HEV IgM was positive.

This patient was successfully managed with Ceftriaxone, Albendazole, Vit.K and adequate IV Fluids over a period of 12 days. The patient was discharged on day 12 of admission. On discharge, he was asymptomatic, the liver functions and the coagulation profile improved considerably and the repeat stool sample didn't show any evidence of round worm.

\section{Discussion}

This case confronts the treating physician with a diagnostic dilemma because of several overlapping clinical features of dengue, leptospirosis, hepatitis $\mathrm{E}$ and Ascariasis. The probable reasons for this triple co-infection in immunocompetent patient could be rapid urbanization, increasing population density, frequent travel, poor water drainage infrastructure and inadequate vector control measures [ 1 ]. It's a well acknowledged fact that multiple infections in an individual can co-exist in this era of AIDS pandemic, however, such an infection is still uncommon in a immunocompetent host . To date, not many cases of mixed infection with dengue and leptospirosis have been described. Only 4 reports of triple co-infections have been reported [ 2 , $9,10,11]$; however, the concurrent rise of leptospirosis during a dengue outbreak has been reported [ 12 ]. The possibility of co-infection with HEV and Ascariasis should also be borne in mind, as water is the vehicle of transmission for HEV, Ascariasis and Leptospira. Most of the available literature shows mixed infection with two or three agents, but concurrent infection with four agents in an immune competent host is being attempted to report for the first time. Quadruple infection of any kind in an immune competent host is a rare entity and a matter of serious concern for the physicians. The under-diagnosis of such cases is very likely due to the overlapping clinical spectrum [ 13 ].

Morbidity and mortality can be quite high in such cases. Our findings attempts to highlight the need for greater awareness of the possibility of mixed infection as well as the need for optimal use of laboratory services to reach a specific diagnosis as early as possible thereby preventing complications.

\section{Message}

This case jolts us, yet again, to the age old problem haunting our errantly growing metropolis which are today devoid of basic sanitation facilities. Moreover expensive and inadequate housing has lead to overcrowding in these cities, where the cities have swollen to an extent where a catastrophe is just waiting to occur. This makes us think that the burden of providing a clean neighbourhood is nothing when compared to the overall burden of infectious diseases borne in India, both in terms of money and morbidity. Our patient was lucky enough to escape this quadruple infection without much life threatening clinical events especially associated with Dengue and Leptospirosis which kills hundreds around India every year. Noteworthy is the fact that all 4 infections described here are conceived from overcrowding and insanitation.

\section{Conflict of Interest}

It is declared that all authors of this report have no conflict of interest.

\section{References}

[1] Comparison between Three Rare Cases of Co-Infection with Dengue, Leptospira and Hepatitis E:Is Early Endothelial Involvement the Culprit in Mortality? RK

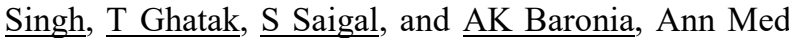
Health Sci Res. 2014 Mar-Apr; 4(Suppl 1): S32-S34

[2] Behera B, Chaudhry R, Pandey A, Mohan A, Dar L, Premlatha MM, et al. Co-infections due to leptospira, dengue and hepatitis E: A diagnostic challenge. J Infect Dev Ctries. 2010;048 - 050.

\section{Volume 4 Issue 11, November 2015}




\section{International Journal of Science and Research (IJSR) \\ ISSN (Online): 2319-7064}

Index Copernicus Value (2013): 6.14 | Impact Factor (2014): 5.611

[4] Bharti AR, Nally JE, Ricaldi JN, Matthias MA, Diaz MM, Lovett MA, Levett PN, Gilman RH, Willig MR, Gotuzzo E, Vinetz JM (2003) Leptospirosis: a zoonotic disease of global importance Lancet Infect Dis. 3: 757771.

[5] Chaudhry R, Premlatha MM, Mohanty S, Dhawan B, Singh KK, Dey AB (2002) Emerging Leptospirosis, North India. Emerg Infect Dis 8: 1526-1527.

[6] BAikat BK, Konar NR, Banerjee G. Hemorrhagic fever in the Calcutta area. (1964) Indian J Med Res 52: 660675.

[7] Gubler DJ (1998) Dengue and Dengue hemorrhagic fever. Clin Microbiol Rev 11: 480-496.

[8] Dar L, Broor S, Sengupta S, Xess I, Seth P (1999) The first major outbreak of dengue hemorrhagic fever in Delhi, India. Emerg Infect Dis 5: 589-590.

[9] Pandey A, Diddi K, Dar L, Bharaj P, Chahar HS, Guleria R, Kabra SK, Broor S (2007) The evolution of dengue over a decade in Delhi, India. J Clin Virol 40: 87-88. .

[10]Rele MC, Rasal A, Despande SD (2001) Mixed Infection due to dengue and leptospira in a patient with pyrexia. Indian J Med Microbiol 19: 206-208.

[11] Levett PN, Branch SL, Edwards CN (2000) Detection of dengue infection in-patients investigated for leptospirosis in Barbados. Am J Trop Med Hyg 62: 112114.

[12] Kaur H, John M (2002) Mixed infection due to leptospira and dengue. Ind J Gastroenterol $21: 206$.

[13] LaRocque RC, Breiman RF, Ari MD, Morey RE, Janan FA, Hayes JM, Hossain MA, Brooks WA, Levett PN (2005) Leptospirosis during dengue outbreak, Bangladesh. Emerg Infect Dis 11:766-769.

[14] Chaudhry R, Pandey A, Das A, Broor S (2009) Infection potpourri: are we watching? Indian J Pathol Microbiol 52:125.

\section{Author Profile}

Dr. Sumit D. Chaudhari is in Department of Medicine, Krishna institute of Institute Medical Sciences, Karad, Maharashtra, India 